\title{
Photo Quiz
}

(For answer and discussion, see page 4421 in this issue [doi:10.1128/JCM.05449-11])

\section{A 53-Year-Old Stem Cell Transplant Recipient with Meningitis and Bacteremia}

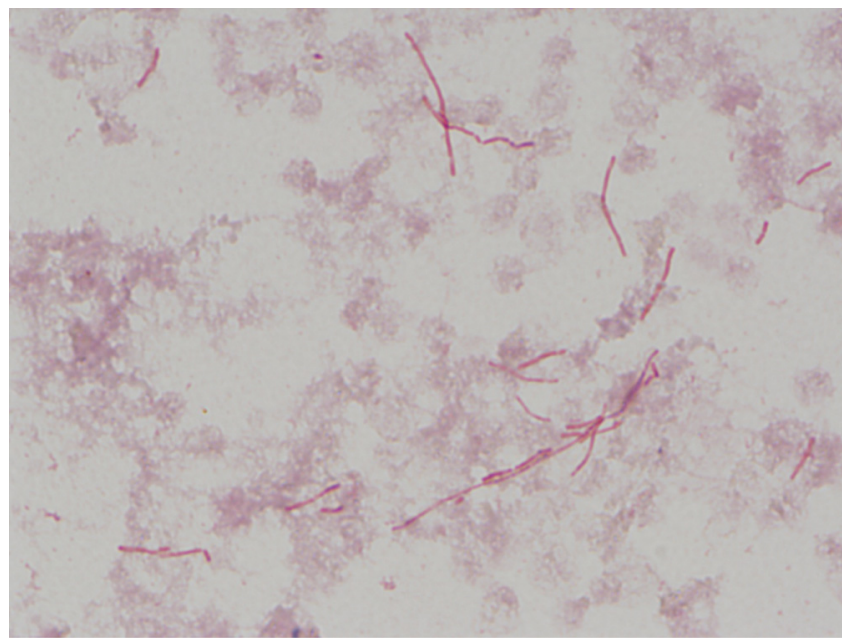

Fig. 1. Gram stain from aerobic blood culture bottle. Magnification, $\times 1,000$ (oil).

A 53-year-old woman with a history of acute lymphocytic leukemia (ALL) was admitted to an outside hospital in Florida. She had received a matched unrelated donor allogeneic peripheral blood stem cell transplant 13 months prior and was on steroid treatment for graft-versus-host disease involving skin, eyes, and liver. She presented with symptoms of bacteremia, subacute left cerebellar infarct with neurological symptoms, and depression secondary to her medical condition. Three sets of blood cultures (Bactec; Becton, Dickinson and Company, Sparks, MD), collected at an outside hospital, were positive (aerobic bottles) for a Gram-negative rod that tested susceptible (MIC $<3 \mu \mathrm{g} / \mathrm{ml})$ to piperacillin-tazobactam but intermediate or resistant to all other antibiotics as determined by a MicroScan Gram-negative panel (Siemens Healthcare Diagnostics, Inc., West Sacramento, CA) and Clinical and Laboratory Standards Institute (CLSI) interpretations for nonEnterobacteriaceae. The patient was treated with piperacillintazobactam for 9 days without improvement. Susceptibility testing was repeated at the outside hospital on a follow-up culture and showed an MIC of $64 \mu \mathrm{g} / \mathrm{ml}$ for piperacillin-tazobactam but no change in the results for the other antibiotics. The patient was subsequently transferred to our tertiary care medical center for further management. Blood cultures (BacT/ Alert; bioMérieux, Durham, NC) were redrawn on admission and again grew a Gram-negative rod in the aerobic bottle that appeared thin and filamentous on initial Gram staining of the broth (Fig. 1). The organism produced pale yellow colonies on sheep blood agar and chocolate agar after overnight incubation at $35^{\circ} \mathrm{C}$ in $5 \% \mathrm{CO}_{2}$. Growth on MacConkey agar was poor. The organism was nonmotile, catalase positive, and oxidase positive. A cerebrospinal fluid specimen collected 4 days after admission also showed Gram-negative rods, some of which were dumbbell shaped and grew the same organism as the blood cultures.

\author{
Hunter Johnson* \\ Eileen M.Burd \\ Emory University School of Medicine \\ 1364 Clifton Rd. NE \\ Atlanta, Georgia 30322
}

Susan E. Sharp

Kaiser Permanente-Northwest 13705 NE Airport Way Portland, Oregon 97230

*Phone: (404) 712-7297

Fax: (404) 727-2519

E-mail: hjohns7@emory.edu 